\title{
ON THE EXISTENCE OF JETS IN THE RECURRENT NOVA T PYXIDIS ${ }^{1}$
}

\author{
T. J. O'BRIEN \\ Astrophysics Research Institute, Liverpool John Moores University, Liverpool L3 3AF, England, UK \\ AND \\ JUDITH G. COHEN \\ Palomar Observatory, Mail Stop 105-24, California Institute of Technology, Pasadena, CA 91125 \\ Received 1997 December 15; accepted 1998 February 25; published 1998 April 7
}

\begin{abstract}
Recently, it has been claimed that the recurrent nova T Pyxidis exhibits oppositely directed jets of ejecta apparent in features seen in $\mathrm{H} \alpha$ emission. Here we demonstrate that these features are in fact emission in the [N II] lines that lie on either side of $\mathrm{H} \alpha$ and arise from the expanding shell associated with this object rather than from collimated jets. We estimate an expansion velocity along a line of sight through the center of the shell of about $500 \mathrm{~km} \mathrm{~s}^{-1}$.
\end{abstract}

Subject headings: circumstellar matter — novae, cataclysmic variables

\section{INTRODUCTION}

T Pyxidis is a recurrent nova with recorded outbursts in 1890 , 1902, 1920, 1944, and 1966 (Webbink et al. 1987). It is notable for possessing a bright nebular shell that has been investigated extensively by Shara et al. (1989, 1997). Recently, Shahbaz et al. (1997, hereafter S97) have presented optical spectroscopy of T Pyx in which they identify emission components redward and blueward of $\mathrm{H} \alpha\left(\mathrm{S}^{+}\right.$and $\mathrm{S}^{-}$, respectively, in their Fig. 1), which they interpret as red- and blueshifted $\mathrm{H} \alpha$ emission from oppositely directed jets. These features occur at 6593 and 6539 $\AA$, respectively, implying line-of-sight velocities of 1380 and $-1082 \mathrm{~km} \mathrm{~s}^{-1}$.

\section{OBSERVATIONS AND DISCUSSION}

On the night of 1997 November 21/22, we obtained several spectra of T Pyx with the Low Resolution Imaging Spectrograph (LRIS) (Oke et al. 1995) on the Keck II telescope in Hawaii. Two $400 \mathrm{~s}$ exposures were made at slit position angles of $30^{\circ}$ and $120^{\circ}$. The 600 grooves $\mathrm{mm}^{-1}$ grating was used, with a slit width of 1.5 matching the seeing. The pixel scale was 0 ".2, and the spectral resolution $8.8 \AA$.

Figure 1 shows a gray-scale representation of the twodimensional spectrum from position angle $30^{\circ}$, clearly revealing the presence of an expanding shell emitting in [N II] and $\mathrm{H} \alpha$ with a signal-to-noise ratio ranging from 10 to 70 . The spectrum from position angle $120^{\circ}$ is similar, although, because the shell is not spherically symmetric (see Shara et al. 1997), there are detailed differences. In order to relate the data to the spectra presented by S97, Figure 1 also includes the spectrum obtained by summing along the slit. Although the spectral resolution is lower than that of S97, it is still obvious that their features $\mathrm{S}^{+}$ and $\mathrm{S}^{-}$are in fact, respectively, the redshifted component of [N II] $\lambda 6583$ and the blueshifted component of [N II] $\lambda 6548$.

Furthermore, by extracting the stellar continuum from our two-dimensional spectrum using Horne's optimal extraction method, available in the software package FIGARO, and then subtracting this from the total spectrum shown in Figure 1, we

\footnotetext{
${ }^{1}$ Based on observations obtained at the W. M. Keck Observatory, which is operated jointly by the California Institute of Technology and the University of California.
}

obtain a reasonable approximation to the shell-only summed spectrum (see Fig. 2). In this spectrum, we have indicated the rest wavelengths of $\mathrm{H} \alpha$ and the two [N II] lines, together with the positions of the blue- and redshifted components arising from the front and back of a shell expanding at a velocity of $530 \mathrm{~km} \mathrm{~s}^{-1}$. It is worth noting that the component at about $6573 \AA$ (arising from a combination of blueshifted [N II] $\lambda 6583$ and redshifted $\mathrm{H} \alpha$ ) is clearly visible in the spectrum in Figure 1 of S97, while the $6555 \AA$ component (from redshifted [N II] $\lambda 6548$ and blueshifted $H \alpha$ ) is blended into the blue wing of $\mathrm{H} \alpha$ in their Figure 1. It is difficult to estimate the expansion velocity from these spectra. Apart from the problems of contamination between $\mathrm{H} \alpha$ and [N II], the shell is clumpy and incomplete. The velocity of $530 \mathrm{~km} \mathrm{~s}^{-1}$ is derived from the wavelength $6595 \AA$ of the redshifted [N II] $\lambda 6583$ line at the point where it crosses the stellar continuum. This corresponds to the expansion velocity along a line of sight through the center of the shell and hence will be independent of the slit position angle. We estimate an uncertainty of $\pm 2 \AA$ on this wavelength, equivalent to $\pm 90 \mathrm{~km} \mathrm{~s}^{-1}$, as a result of the contamination by the stellar continuum and of the spectral resolution. A better estimate would require more kinematical data at higher resolution across the whole shell and a plausible model for its structure. Note that the summed spectrum shown in Figure 2 peaks shortward of $6595 \AA$ because this particular feature is dominated by emission from a bright part of the shell $2^{\prime \prime}-3^{\prime \prime}$ below the star (see Fig. 1), which is at lower radial velocities.

In conclusion, we suggest that there is little evidence to support the existence of collimated jets in T Pyx. However, the data are consistent with the presence of a shell expanding at about $500 \mathrm{~km} \mathrm{~s}^{-1}$ and emitting more strongly in [N $\left.\mathrm{II}\right]$ than in $\mathrm{H} \alpha$; this is in broad agreement with the findings of Shara et al. (1989). However, a more detailed modeling of the structure of the shell and further higher spectral resolution observations are required in order to reconcile this line-of-sight expansion velocity with the upper limit on the velocity in the plane of the sky of $40 \mathrm{~km} \mathrm{~s}^{-1}$ derived from Hubble Space Telescope observations of the proper motion of knots in the nebular shell (Shara et al. 1997).

We are grateful to M. Shara and C. Gill for useful discussions and to an anonymous referee for helpful comments on the 


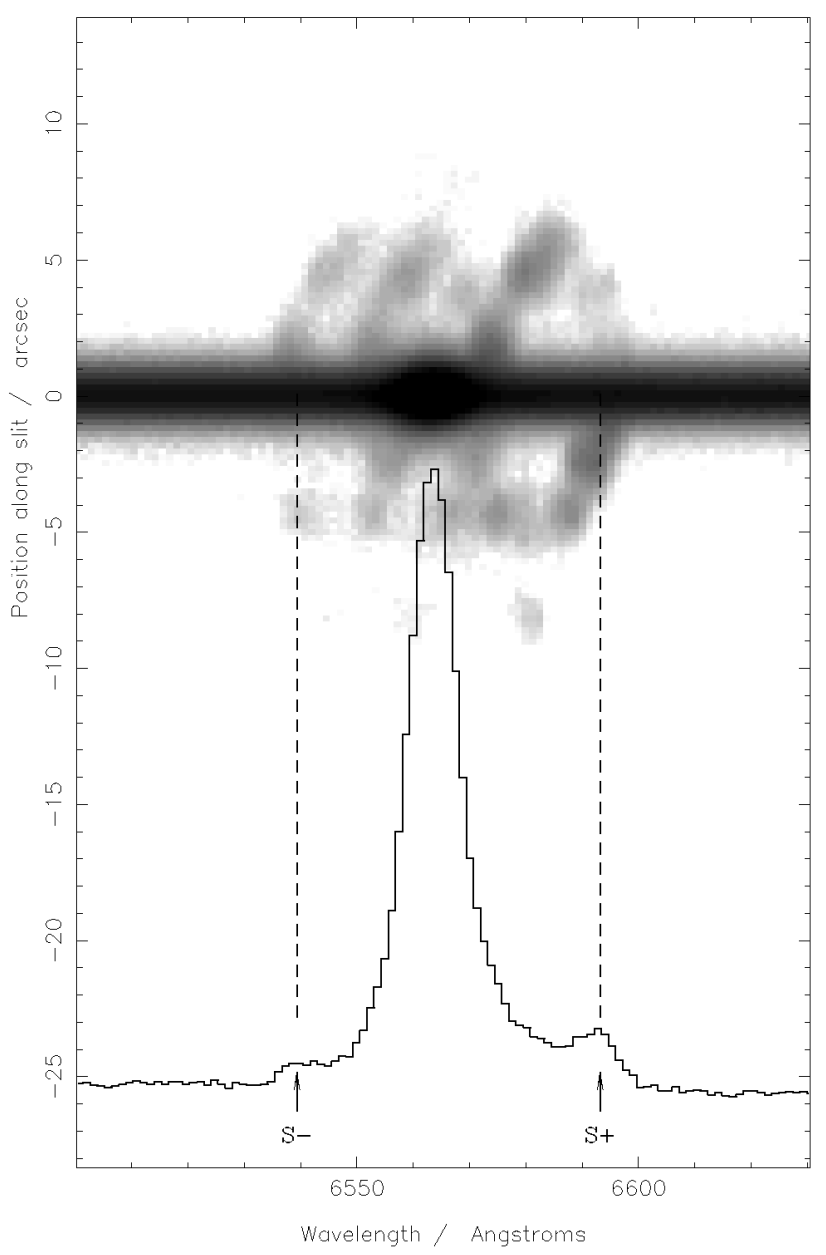

FIG. 1.-The two-dimensional spectrum of T Pyx plotted as a logarithmic gray scale. Below it is the one-dimensional spectrum obtained by summing in the spatial direction. The features referred to by Shahbaz et al. (1997) as $\mathrm{S}^{+}$and $\mathrm{S}^{-}$are also indicated (at the wavelengths taken from their paper), as is their origin in the [N II] lines from the expanding shell.

original version of this Letter. The entire Keck/LRIS user community owes a huge debt to Jerry Nelson, Gerry Smith, Bev

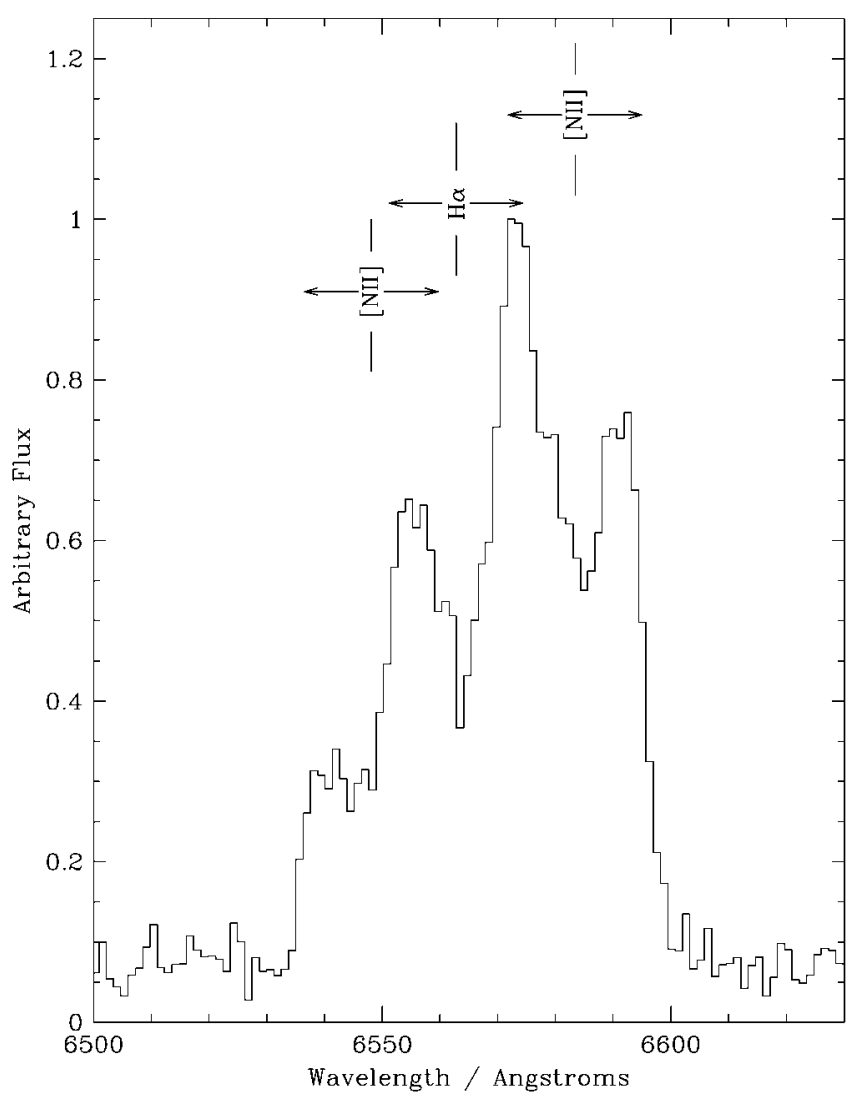

FIG. 2.-The spectrum of the shell obtained by subtracting the stellar contribution from the original data shown in Fig. 1. The rest wavelengths of $\mathrm{H} \alpha$ and the two [N II] lines are indicated, as are the wavelengths to which these lines would be red- and blueshifted by an expansion along the line of sight of $530 \mathrm{~km} \mathrm{~s}^{-1}$

Oke, and many other people who have worked to make the Keck telescope and LRIS a reality. We are grateful to the W. M. Keck Foundation, and particularly its late president, Howard Keck, for the vision to fund the construction of the W. M. Keck Observatory.

\section{REFERENCES}

Oke, J. B., et al. 1995, PASP, 107, 375

Shahbaz, T., Livio, M., Southwell, K. A., \& Charles, P. A. 1997, ApJ, 484, L59 (S97)

Shara, M. M., Moffat, A. F. J., Williams, R. E., \& Cohen, J. G. 1989, ApJ, 337,720
Shara, M. M., Zurek, D. R., Williams, R. E., Prialnik, D., \& Gilmozzi, R. 1997, AJ, 114, 258

Webbink, R. F., Livio, M., Truran, J. W., \& Orio, M. 1987, ApJ, 314, 653 